\title{
Coordinated Pitch \& Torque Control of Large-Scale Wind Turbine Based on Pareto Eciency Analysis
}

\author{
Lin, Zhongwei ; Chen, Zhenyu; Wu, Qiuwei; Yang, Shuo ; Meng, Hongmin
}

Published in:

Energy

Link to article, DOI:

10.1016/j.energy.2018.01.055

Publication date:

2018

Document Version

Peer reviewed version

Link back to DTU Orbit

Citation $(A P A)$ :

Lin, Z., Chen, Z., Wu, Q., Yang, S., \& Meng, H. (2018). Coordinated Pitch \& Torque Control of Large-Scale Wind Turbine Based on Pareto Eciency Analysis. Energy, 147, 812-825. https://doi.org/10.1016/j.energy.2018.01.055

\section{General rights}

Copyright and moral rights for the publications made accessible in the public portal are retained by the authors and/or other copyright owners and it is a condition of accessing publications that users recognise and abide by the legal requirements associated with these rights.

- Users may download and print one copy of any publication from the public portal for the purpose of private study or research.

- You may not further distribute the material or use it for any profit-making activity or commercial gain

- You may freely distribute the URL identifying the publication in the public portal 


\title{
Coordinated Pitch \& Torque Control of Large-Scale Wind Turbine Based on Pareto Efficiency Analysis ${ }^{\text {th }}$
}

\author{
Zhongwei Lin ${ }^{\mathrm{a}, *}$, Zhenyu Chen ${ }^{\mathrm{a}}$, Qiuwei Wu ${ }^{\mathrm{b}}$, Shuo Yang ${ }^{\mathrm{c}}$, Hongmin Meng ${ }^{\mathrm{a}}$ \\ ${ }^{a}$ State Key Laboratory of Alternate Electrical Power System with Renewable Energy Sources, \\ School of Control and Computer Engineering, \\ North China Electric Power University, Beijing 102206, P. R. China \\ ${ }^{b}$ Center for Electric Power and Energy, Department of Electrical Engineering, \\ Technical University of Denmark, Kgs. Lyngby 2800, Denmark \\ ${ }^{c}$ State Key Laboratory of Operation and Control of Renewable Energy and Storage Systems, \\ China Electric Power Research Institute, Beijing 100085, P. R. China
}

\begin{abstract}
For the existing pitch and torque control of the wind turbine generator system (WTGS), further development on coordinated control is necessary to improve effectiveness for practical applications. In this paper, the WTGS is modeled as a coupling combination of two subsystems: the generator torque control subsystem and blade pitch control subsystem. Then, the pole positions in each control subsystem are adjusted coordinately to evaluate the controller participation and used as the objective of optimization. A two-level parameters-controllers coordinated optimization scheme is proposed and applied to optimize the controller coordination based on the Pareto optimization theory. Three solutions are obtained through optimization, which includes the optimal torque solution, optimal power solution, and satisfactory solution. Detailed comparisons evaluate the performance of the three selected solutions and provide the optimized controller coordination suggestions according to different requirements.
\end{abstract}

Keywords: WTGS, Coordination, Pareto efficiency, Multi-objective optimization

\footnotetext{
This work is partially supported by the National Basic Research Program of China (973 Program) (Grant No. 2012CB215203), the State Grid Corporation of China Science and Technology Project: Research and application of key technologies for active support and coordination control of renewable energy generation, the National Natural Science Foundation of China (No. 61203043, No. 51036002) and the Fundamental Research Funds for the Central Universities.

${ }^{*}$ Corresponding author

Email address: lzw@ncepu.edu.cn (Zhongwei Lin)
} 


\section{Introduction}

In recent years, with rapid growth of wind power, modern wind turbines(WTs) have been developed with larger-scale blades and capacity, which brings more challenges to the traditional control. The wind turbine generator system(WTGS) works mainly in two separate regions: maximize wind energy conversion efficiency below the rated wind speed [1], or maintain the output power at the rated power above the rated wind speed $[2,3,4]$. There exist corresponding strategies to achieve smooth transition or stabilized output $[5,6,7,8]$. Motivated by the development of control theory, the existing WT control strategies mainly combine the proportional, integral and derivative(PID) control, linear control or nonlinear control [2, 9, 10, 11, 12]. Most practical WT control still adopts the PID control, especially the PI control. The related research mainly focuses on proposing advanced PID control strategies by introducing more related variables, such as $[11,13]$.

The linear state-space model is usually adopted for WTGS based on the operatingpoint linearization. The state-space representation can better represent the relationship between different system states, and the zero-poles can also be easily calculated, which is essential for the analysis of system response and stability. Moreover, the zero-poles of the system can be configured by state feedback or output feedback, and state parameters can also be easily obtained by the state observer. Several state-space models have been proposed to describe the WTGS from different aspects. [14] introduced several WTGS state-space models with different orders and state variables when the requirements and control objects are different. [15] and [16] investigated the multi-objective optimal control by applying the pole placement technique, where [15] tried to place the poles of the WTGS state-space model into specific LMI regions. Since the WTGS is a nonlinear system, a lot of work has applied the nonlinear control to the WT control, such as the feedback linearizing control, back-stepping control and sliding mode control, etc. [10, 17, 18, 19]. For the nonlinear WTGS model, it is still possible to regulate the dynamic response by combining the geometric linearization and pole placement techniques [19].

From the optimal control perspective, the model order of the WTGS is closely related to the control objectives. Several high-order models for accurate representation of the WTGS were proposed in [6, 7, 8, 20]. Especially, [6] described the WTGS with a set of differential equations to analyze the model sensitivity related to the wind speed. For the convenience of practical applications, a three-order WTGS state-space model is adopted in this paper. The wind turbine control system can be divided into two parts: the torque \& power controller and the blade pitch controller. Normally, the control strategies combine both controllers to consider the WT as a multi-input system, and design the controller accordingly when the wind speed is 
above the rated speed $[14,16,17]$. The torque \& power controller is usually set to the constant power mode, and the generator power is kept constant so that the generator torque response is inversely proportional to the generator speed, where the blade pitch controller is the only actuator used to adjust the wind energy captured. Some research investigated the control of both generator torque and blade pitch, usually modeling two controllers in a state-space model and controlling the system by the regional pole placement method or robust control method [15, 19, 21]. The system diagram of the traditional methods is shown in Figure 1. Such methods can improve the coordination of multiple controllers and use the generator torque $\&$ power controller to assist the pitch actions. However, the involvement degree of each controller is hard to control especially in the regional pole placement method. Therefore, it is necessary to investigate the role of torque \& power controller precisely that coordinately work with the blade pitch controller to improve the control effectiveness and achieve better responses.

It is worth mentioning that, during the coordination analysis, more than one variable need to be considered, which leads to a multi-objective optimized problem, i.e., find the most important variables need to be considered and strike a balance between different objectives. Several research studied the multi-objective optimization problems. [22] introduced several multi-objective optimization methods, and [23] showed a typical application of multi-objective optimization in multi-regional electricity systems. The general optimization method usually minimizes a cost function, however, the acquisition of the cost function is subjective. [24, 25] proposed another method to solve the problem and optimized the weight matrix in model predictive control: by using the Pareto theory to qualitatively analyze two objectives and obtain a satisfactory solution by calculating the gradient. However, it is still meaningful to further analyze the controller coordination for the WTGS by optimizing the pole position, due to the essential characteristic with the high-order multi-inputs and multi-objectives.

In this paper, the WTGS is linearized into a three-order linear state-space model (the detailed linearization process is in the Appendix), in which the torque and pitch controllers are separated into two subsystems: the generator torque \& power control system and blade pitch control system. Poles of the two subsystems are precisely placed to control the WT. Simulations were carried out with different poles position to compare and evaluate the performance. Generator power, generator torque and blade pitch movement are the objective variables, which need to be optimized in the controller coordination. Multi-objective optimization strategies and Pareto efficiency are used to analyze the responses of the controller coordination and objective variables balanced solution. The system structure diagram is shown 
in Figure 2, and the coordinated solution will be evaluated in the time domain, eigenvalues and frequency domain.

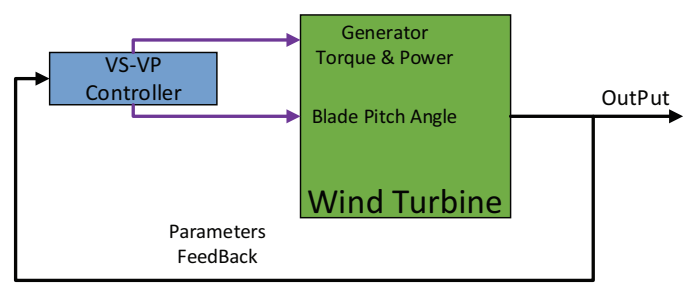

Figure 1: Classic System Structure Diagram

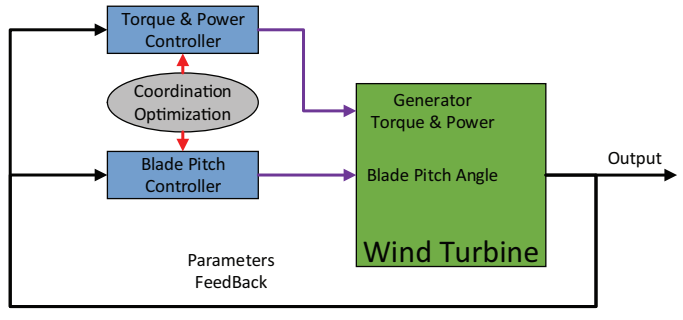

Figure 2: System Structure Diagram Proposed

The rest of the paper is organized as follows: In Section 2, the two-level parameterscontrollers coordinated optimization system is described. In Section 3, the definitions related to Pareto efficiency are introduced and adopted for the analysis of several satisfactory solutions. In Section 4, the control effectiveness of different solutions is evaluated from the aspects of time domain responses, eigenvalues and frequency domain, respectively. Section 5 gives the poles placement suggestions for different conditions such that the controllers are coordinated for different control objectives. Section 6 concludes this paper.

\section{Two-Level Parameters-Controllers Coordination}

Many parameters of the WTGS can be measured and have an important impact on the wind turbine characteristic. In this section, the analysis is carried out based on the pole movement of both subsystems, where three parameters are selected as the optimization objective and forms a two-level parameters-controllers coordinated optimization system, i.e., the parameters coordination optimization for each controller as the first level, and the controller coordination as the second level.

To evaluate the influence of each subsystem, several simulations are simulated under turbulent wind with mean speed $15 \mathrm{~m} / \mathrm{s}$ and turbulence intensity 12. Ten DOFs (Degree of Freedom) including the tower fore-aft, flexible blade and flexible drive-train are used in the simulations, which is aimed at good control of the highorder WT model with the coordinated controllers designed from a third-order model. A higher-order model is quite difficult to be used in the industrial field, while the lower-order one is more useful for industrial processes. During simulations, the natural frequency and damping ratio in both subsystems remain consistent to avoid the unnecessary interference. In the meantime, the single pole on the right hand side is 
placed in an appropriate interval to keep the wind turbine stable and get the corresponding response data for the further evaluations. The single pole in the torque control subsystem changes from -0.5 to -0.05 , and the single pole in the pitch control subsystem changes from -2.05 to -0.9 . Since the natural frequency and damping ratio keep consistent, the second order part in the denominator of the transfer function will remain unchanged and keep two poles distributed symmetrically without any movement. All poles movement mentioned in the rest of this paper refers to the single one on the right hand side in both subsystems, and are called as pitch-pole and torque-pole for convenience. The poles map is shown in Figure 3.

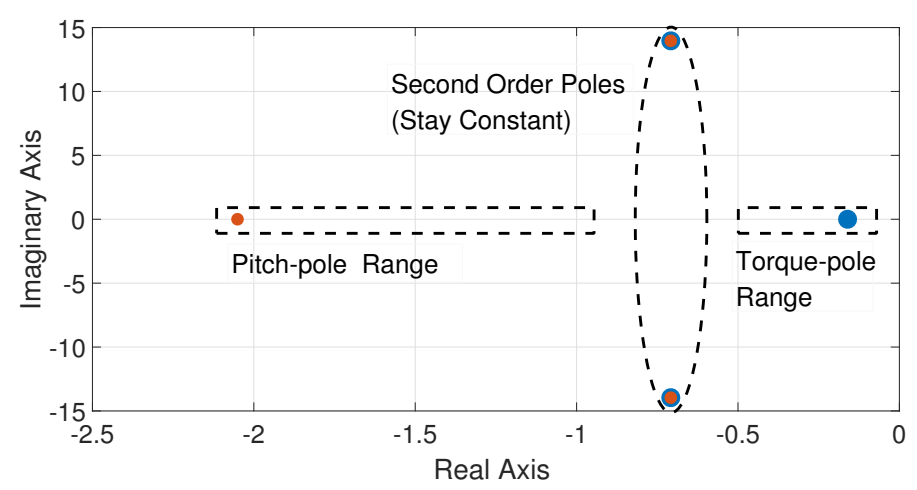

Figure 3: Poles Map

The analysis of the pitch control subsystem and torque control subsystem are carried out based on series of data. During simulations, the operating point is set as the rated power condition, where the limited power scenario is not considered in this paper. Four most important parameters are analyzed to evaluate the responses, which are the generator power output, generator speed, generator torque the blade pitch movement. In order to evaluate the output stability and fluctuation, all four parameters are calculated as standard deviation (STD) and analyzed based on the pole position in both the torque and pitch control subsystems. All values in the curves are normalized with the corresponding response values obtained by an industrial baseline controller designed for the specific wind turbine, which is developed by NREL and provided in FAST $[5,26]$. The normalized values are obtained by $\frac{\psi_{\text {Self }}}{\psi_{B C}}$, where the $\psi_{\text {Self }}$ are the respective response parameters with the pole-placed statefeedback controller proposed in this paper, and $\psi_{B C}$ are the respective response parameters with the baseline controller. Obviously, when the ordinate of one point on the STD curve is less than 1, it implies a more stable output than the baseline control with the corresponding parameters. 
The response data are analyzed based on the pole movement in both the pitch and torque control subsystems, which are illustrated in Figure 4.
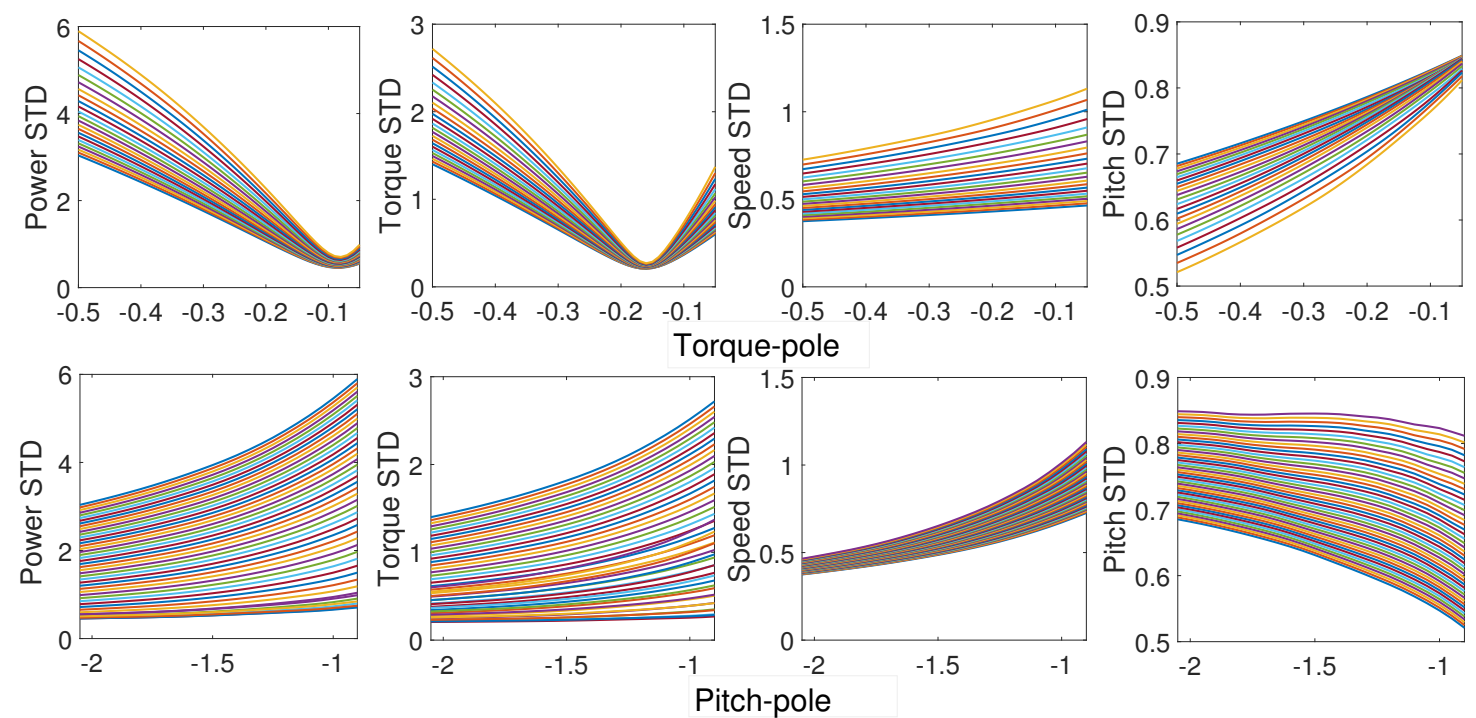

Figure 4: Pole Movement (Figures on the top-layer are the responses shown with the torque-pole positions as x-axis, where lines with different colors shows the different responses with different pitch-pole. Figures on the bottom layer are the responses with the pitch-pole as x-axis)

Obviously, when the pitch-pole is moving away from the imaginary axis, the generator power, torque and speed STD decrease but the pitch movement STD increases. Clearly, the blade pitch movement increases with the pitch-pole moving away from the imaginary axis, which means that the blade pitch action is more sensitive to the wind turbulence and leads to a more stable output, but the improvement of the output stability is at the expense of the pitch movement. Responses with the torque-pole movement are more interesting. With the torque-pole moving away from the imaginary axis, the blade pitch movement STD and generator speed STD are decreased gradually, but the generator power and generator torque show very different responses. Both generator power and generator torque are becoming stable at first, their STDs reach the minimum point and then increase rapidly, and the minimum points of the generator power STD and generator torque STD are surprisingly different. The generator power STD reaches its minimum point at first, and the generator torque STD keeps decreasing while the generator power STD increases till the minimum point of generator torque STD. The further movement of the torque-pole will increase both the generator power and torque STD, in another word, the output quality is getting worse. 
The above analysis shows that, it is difficult to find the optimal poles position for both the pitch and torque control subsystems. Three most important parameters need to be balanced in controller coordinated optimizations: the generator power stability, generator torque stability and blade pitch movement, or in another word: the energy output quality, blade root loads caused by the blade pith frequent movement, and drive shaft loads caused by the shaft torque fluctuation. In the pitch control subsystem, a more stable power output will cost more frequent blade pitch actions, and how to coordinate the generator power and torque is the problem to be solved in torque control subsystem. Thus, a two-level parameters-controllers coordinated optimization system is proposed to optimize the controller coordination: The parameter-coordination is the first level for the satisfactory controllers, i.e., the generator torque/power coordination in the torque controller, and the generator power/pitch movement coordination in the pitch controller. After parametercoordination optimization, one or several satisfactory controller combinations can be achieved and form the controller coordination solutions. The system structure is shown in Figure 5.

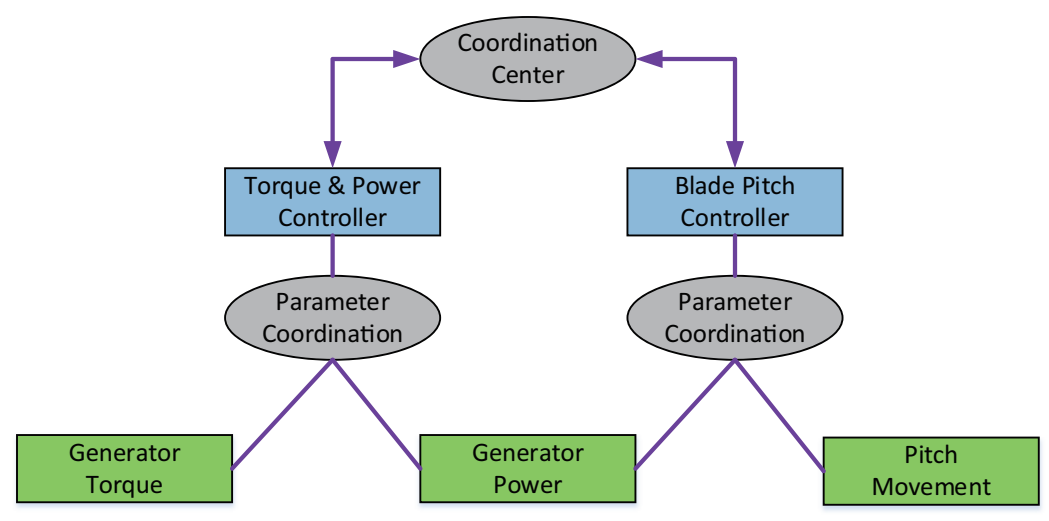

Figure 5: System Tree Structure

\section{Coordinated Control Design through Pareto and Pole Placement}

In this section, the multi-objective coordinated optimization techniques are applied on the torque and pitch control subsystems to obtain an optimal or a regional optimal pitch-pole and torque-pole at $15 \mathrm{~m} / \mathrm{s}$ wind speed operating point.

According to the previous discussion, three main variables are involved during the pole optimization. For the multi-objective problem, several optimization strategies first form a cost function with weights for different objectives, then solve the optimization problem with the cost function. [24, 25] introduced an optimization 
strategy based on Pareto efficiency to optimize the two-objective optimization problem based on the model predictive control. In this paper, the optimization problem has been divided into two parts and each part deals with two parameters. The fundamental concepts of the multi-objective (MO) optimization, definitions of Pareto optimal solutions and Pareto efficiency are given as follows to solve this optimization problem. More details can be referred to [22, 24, 27].

Definition 1 (Optimization Problem) Assuming two objective variables marked as $\alpha, \beta$ and the decision variable $W \in \Psi$, where $\Psi$ is the feasible set, find the optimal solution $W_{\text {opt }}$ for two objective values $\alpha_{\text {opt }}, \beta_{\text {opt }}$, in which $W \in \Psi, \alpha \in A, \beta \in B$, and $A$ and $B$ are constraints for each objective variable.

\subsection{Pareto Efficiency}

In contrast to the single-objective optimization problem, there may exist no global solution, which is optimal for all contradicting objectives in the multi-objective optimization problem; it is hence desirable to seek a set of good solutions that are not worse than other solutions. This set of solutions is called the Pareto optimal solutions to the MO problem perspective. These solutions are also termed non-inferior, admissible, or efficient solutions.

Definition 2 (Pareto Efficiency) Pareto efficiency or Pareto optimally is a state of allocation of resources, from which it is impossible to reallocate to make any one individual or preference criterion better off without making at least one individual or preference criterion worse off.

Definition 3 (Dominance) Given two solutions $W_{1} \in \Psi$ and $W_{2} \in \Psi$ for the two objective values $\left(\alpha_{1}, \beta_{1}\right)$ and $\left(\alpha_{2}, \beta_{2}\right)$ of the MO problem, respectively, $\left(\alpha_{1}, \beta_{1}\right)$ is said to dominate $\left(\alpha_{2}, \beta_{2}\right)$, if $\left(\alpha_{1}<\alpha_{2}\right)$ and $\left(\beta_{1}<\beta_{2}\right)$.

Definition 4 (Pareto Optimal Solution) A solution $W^{*}$ is the Pareto optimal solution of the MO problem with the respect to $\Psi$, if there does not exist another feasible solution $W^{o} \in \Psi$ such that the objective values $\left(\alpha^{o}, \beta^{o}\right)$ dominate $\left(\alpha^{*}, \beta^{*}\right)$.

Definition 5 (Pareto Improvement) A Pareto improvement is a change to a different allocation that makes at least one individual or preference criterion better off without making any other individual or preference criterion worse off, given a certain initial allocation of goods among a set of individuals. An allocation is defined as "Pareto efficient" or "Pareto optimal" when no further Pareto improvements can be made, in which case we are assumed to have reached Pareto optimally.

Definition 6 (Weak Pareto efficiency) A "weak Pareto optimum" (WPO) is an allocation, for which there are no possible alternative allocations whose realization would cause every individual to gain. Thus, an alternative allocation is considered to be a Pareto improvement if and only if the alternative allocation is strictly preferred 
by all individuals. When contrasted with weak Pareto efficiency, a standard Pareto optimum as described above may be referred to as a "strong Pareto optimum" (SPO).

Definition 7 (Pareto Frontier) Consider a system with function (mapping) $f$ : $\mathbb{R}_{n} \rightarrow \mathbb{R}_{m}$, where $X$ is a compact set of feasible decisions in the metric space $\mathbb{R}_{n}$, and $Y$ is the feasible set of criterion vectors in $\mathbb{R}_{m}$, such that $Y=\left\{y \in \mathbb{R}_{m}: y=\right.$ $f(x), x \in X\}$. Assume that the preferred directions of criteria values are known. A point $y^{\prime \prime}$ is preferred to (strictly dominates) another point $y^{\prime}\left(y^{\prime \prime}\right.$ and $\left.y^{\prime} \in \mathbb{R}_{m}\right)$, written as $y^{\prime \prime} \succ y^{\prime}$. The Pareto frontier $P(Y)$ is thus written as:

$$
P(Y)=\left\{y^{\prime} \in Y:\left\{y^{\prime \prime} \in Y: y^{\prime \prime} \succ y^{\prime}, y^{\prime \prime} \neq y^{\prime}\right\}\right\} .
$$

The typical example of a pareto frontier is shown as follows.

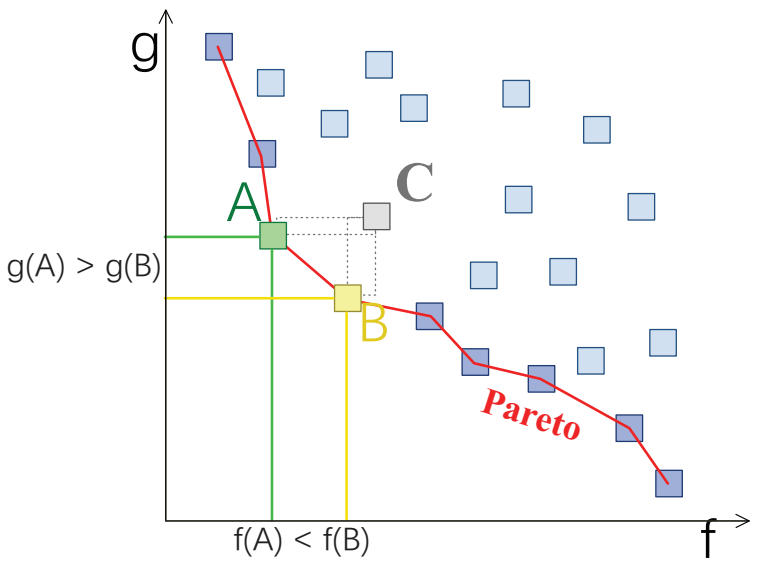

Figure 6: Pareto Frontier

The boxed points represent feasible choices, and smaller values are preferred to larger ones. Point $\mathrm{C}$ is not on the Pareto frontier because it is dominated by both point $\mathrm{A}$ and point $\mathrm{B}$. Points $\mathrm{A}$ and $\mathrm{B}$ are not strictly dominated by any other, and hence do lie on the frontier.

\subsection{Analysis of The Satisfactory Torque-pole}

Similar to Figure 4, the response data are plotted with the generator torque STD as $\mathrm{x}$-axis and generator power STD as y-axis, to select an appropriate torque-pole position that balance the torque and power output, which is shown in Figure 7. 

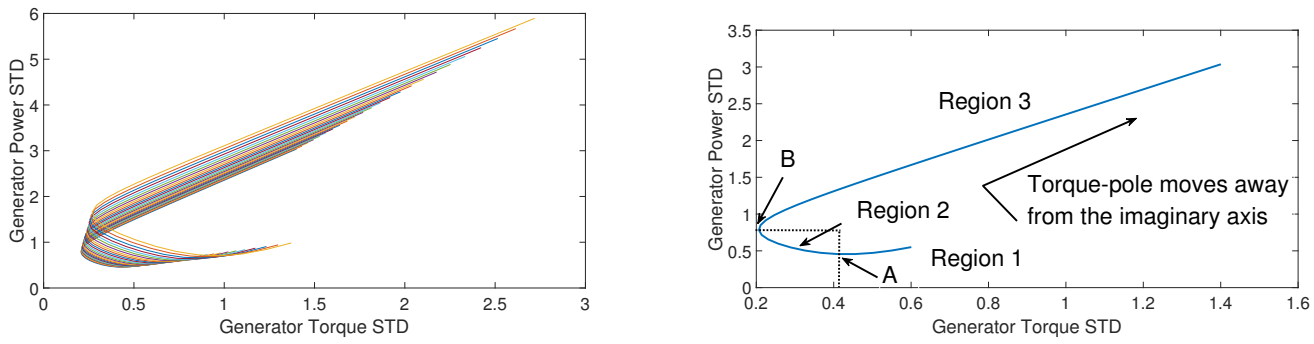

Figure 7: Torque-Power Coordination Curves (Lines in left figure with different colors stand for the simulations with different pitch-pole. As the line moves, the torque pole changes gradually)

In the left of Figure 7, it can be found that although the pitch-pole changes the positions and curve shapes, the trend remains the same, thus the curve at the front is shown in the right of Figure 7 as an example for convenience.

In the right of Figure 7, the curve can be further divided into three regions. When the torque-pole moves away from the imaginary axis, the torque-power curve passes through regions 1, 2, 3 in sequence. Similar situations exist in regions 1 and 3: the generator power STD and generator torque STD keep the same change trend following the movement of the torque-pole, i.e., both the generator torque STD and generator power STD decrease with the torque-pole moving away from the imaginary axis in region 1 and increase in region 3, which makes point $\mathrm{A}$ the most dominant one in region 1 and point $\mathrm{B}$ in region 3 correspondingly. In region 2, the generator power STD increases when the generator torque STD decreases, in other words, the two parameters cannot be improved simultaneously, and the dominance of one part is at the expense of another deterioration. Therefore, possible solutions in region 2 are on the Pareto frontier that cannot guarantee any Pareto improvement. A satisfactory solution should be obtained through optimization methods on the Pareto frontier.

It is important to note that, a satisfactory solution is different from an optimal solution, when the different objectives are difficult even impossible to converge to the same point while the strong Pareto optimal can be obtained. Generally, several objectives reach a Pareto frontier and every solution on the frontier are optimal solutions (weak Pareto optimum). However, a high-level optimization objective is required to analyze the difference between optimal solutions and obtain the only one that satisfies the high-level optimization objective. The satisfactory solution is a weak Pareto optimal condition. 

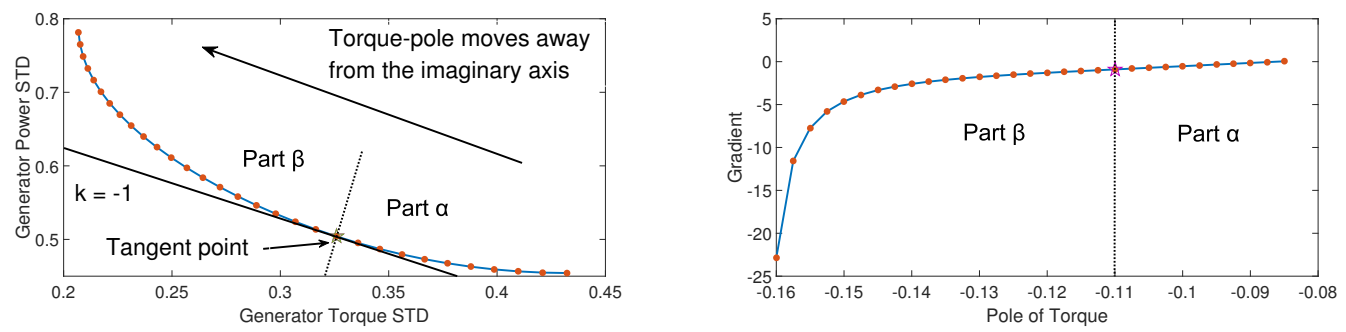

Figure 8: Torque-Power Coordination Curves in Region 2 and Corresponding Gradients

The curve in region 2 is extracted and shown in Figure 8, and the gradient at each data point in region 2 is calculated. Obviously, the gradient at each point remains negative, and the gradual decrease of its absolute value shows that the rate of decrease of the generator power STD is continuously reduced with the increase of the generator torque STD. Take the generator torque STD as investment and the reduction of the generator power STD as profit, then the profit will be lower with the increase of per unit investment. The trend is still the same if swapping the investment and profit. The tangent point of the curve with a line slope of -1 is shown in this figure. The slope of the change at the tangent point in generator power STD and generator torque STD is the same, and region 2 is divided into two parts: Part $\alpha$ (between the generator power STD minimum point and the tangent point), where the stability of generator power is more important than the generator torque; and part $\beta$ (between the tangent point and the generator torque STD minimum point), where the stability of the generator torque is more important than generator power. The figure of gradient shows the focus in the two parts. The data point with the star is the tangent point on the left side figure, the gradient at the tangent point is greater than one, and the gradient of the previous points is less than one, corresponding to part $\beta$ in the left figure, in which the generator power STD increases faster than the generator torque STD declines, and part $\alpha$ is the opposite.

The satisfactory torque-pole can be obtained at the $15 \mathrm{~m} / \mathrm{s}$ wind speed operating point, and is -0.11 at the tangent point. Since previous analysis only used on a single curve with a specific pitch-pole for the satisfactory pitch-pole, the same strategy is used on the other curves with different pitch-pole. The results show that all these curves have the same tangent point with the torque-pole at -0.11 . Thus it can be concluded: The satisfactory torque-pole at $15 \mathrm{~m} / \mathrm{s}$ wind speed is -0.11 considering the balance between generator power and torque with different pitch-poles. Figure 4 shows that both of the generator speed stability and pitch action are improved when the torque-pole moves from the minimum point of generator power to the one of generator torque. Further analyse of the controller coordination will discuss this 
problem with more details.

\subsection{Analysis of The Satisfactory Pitch-pole}

The satisfactory torque-pole has already been obtained. In this subsection, the satisfactory pitch-pole is discussed to balance the generator power stability/blade pitch movement in the pitch control subsystem with the satisfactory torque-pole.
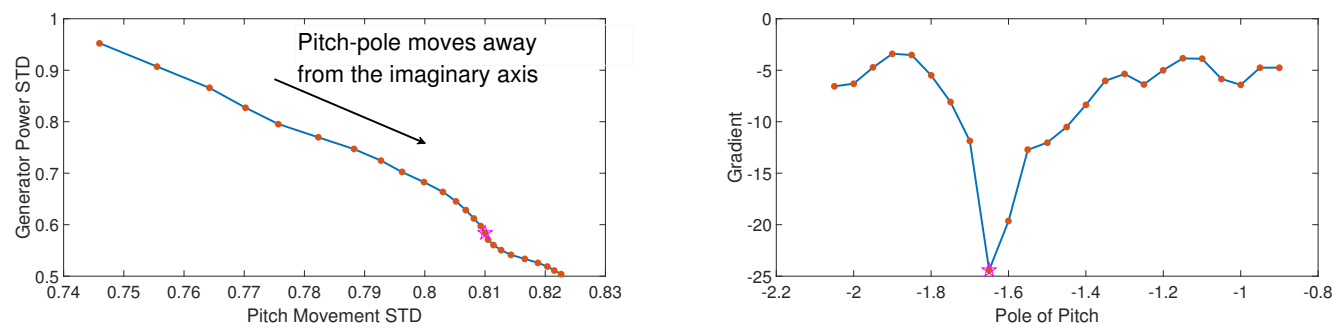

Figure 9: Pitch-Power Coordination Curves and Corresponding Gradients

In Figure 9, take the pitch movement STD as x-axis and generator power STD as y-axis, the gradient at each point is also calculated and plotted. With the pitchpole moving away from the imaginary axis, the gradient curve remains negative, the pitch movement keeps increasing and the generator power keeps decreasing in the meantime. Clearly, the gradient curve is in a state of flux, but a minimum point is very clear and marked with a star, which shows the fastest decline point in the pitch-power curve. It means that the generator power STD decreases fastest in the curve when pitch movement grows very little and is chosen as the satisfactory point. Moreover, the current pitch-pole is the satisfactory solution.

\subsection{Optimal Conditions in Torque-Power Coordination}

Data in Figure 7 including the Pareto frontier are shown in Figure 10 in the form of scatter data points. 


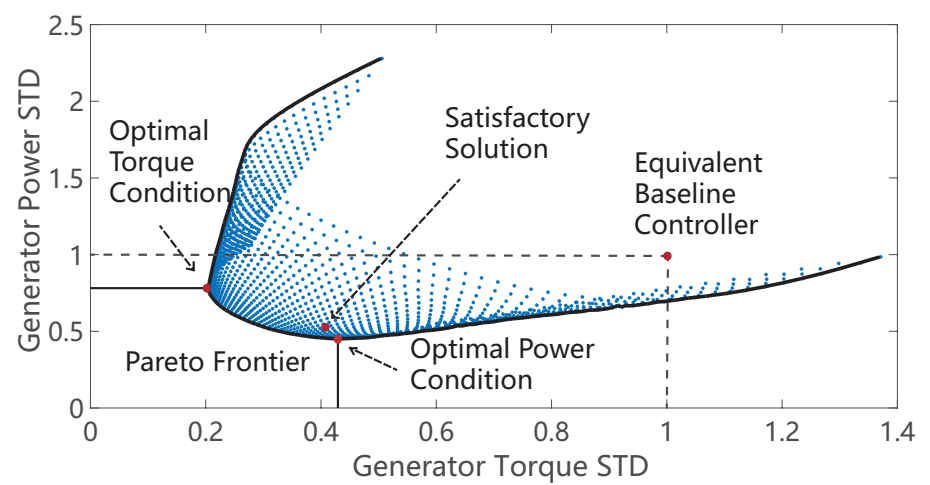

Figure 10: Pareto Frontier for Torque-Power Coordination

Similar to Figure 7, Figure 10 can be also divided in three regions. Clearly, the minimum point of generator power STD is the optimal power condition, and the minimum point of generator torque STD is the optimal torque condition. The intermediate region between two optimal conditions is a typical Pareto Frontier and two optimal conditions are its initial and terminal points. Coincidentally, all points on the Pareto Frontier have the same pitch-pole at -2.05 .

Note that, the above sections analyze torque-power curves separately and reach a same satisfactory torque-pole with different pitch-pole, then obtain the satisfactory pitch-pole. Discussions in this subsection are based on all data, which makes the optimal power and torque conditions as the global optimal solution. Generally speaking, three pairs of poles are obtained in the previous discussions. Take the torque-pole as abscissa and the pitch-pole as ordinate, then the optimal power solution is $(-0.08,-2.05)$, the optimal torque solution is $(-0.16,-2.05)$ and the satisfactory solution is $(-0.1,-1.65)$.

The solution equivalent to the baseline controller and the satisfactory solution are marked in Figure 10, through which it can be seen that the satisfactory solution is slightly off the Pareto frontier of generator power and generator torque (to balance the output and blade pitch movement). The satisfactory solution is not far from the optimal power solution, which indicates that the choice of the satisfactory solution is acceptable in all situations. On the other hand, the solution equivalent to the baseline controller is far away from the Pareto frontier in both generator torque and generator power, which explains that the ability of blade pitch angle movement has not been fully utilized in the baseline controller, and a better control effect can be achieved by increasing the blade pitch sensitivity. Whether such improvement in power stability leads to an increase in load, the analysis of blade loads and drive shaft loads will be given in the next section. 


\section{Effectiveness Evaluation}

Several solutions have been obtained through the previous analyse. In this section, the effectiveness and improvement are evaluated to make sure the selected poles will give a better response than the conventional baseline control strategy, especially in load conditions. The responses are analyzed in the time domain, eigenvalues, and the frequency domain, respectively.

\subsection{Time Domain Evaluation}

Several parameters are shown in histogram figures, response curves and spider diagrams as follows to evaluate the performance improvement. The analysis can be divided into three parts: the basic analysis, drive train loads analysis and blade loads analysis. Output stability and pitch actions are the main variables in the basic analysis. Besides, three bending moments on the blade root are analyzed to evaluate the blade loads, and three thrust forces on the low-speed shaft are used to evaluate the drive shaft loads. The load parameters and its abbreviations are listed in Table 1. Some other load parameters like the blade edge-wise and flap-wise moments are adopted in $[28,29]$, but these parameters are just the mapping of bending moments under the rotating coordinate system into the non-rotating coordinate system and are basically the same. The load parameters considered in this paper are all based on the coordinate system that rotates with the rotor or the drive shaft. Specific definitions about load parameters and corresponding coordinate systems can be referred to [5].

Table 1: Load Parameters

\begin{tabular}{|l|l|}
\hline RootMxc & The blade in-plane moment \\
\hline RootMyc & The blade out-of-plane moment \\
\hline RootMzc & The blade pitching moment \\
\hline LSSFxa & Low-speed shaft thrust force \\
\hline LSSFya & Rotating low-speed shaft shear force along ya-axis \\
\hline LSSFza & Rotating low-speed shaft shear force along za-axis \\
\hline
\end{tabular}

The response curves of four basic parameters are plot in Figure 11. In all the following response curves, the blue curves represent the baseline controller and the orange curves represent the satisfactory solution. Since the difference between the three solutions in the response curves is not clear enough to be distinguished in one figure, the responses of the optimal power and optimal torque solutions are not plotted in curves, and will be analyzed in histograms. In the histograms of Figure 12, the blue column is the corresponding response parameter of the baseline controller, 
and the columns in red, green and purple stand for the satisfactory condition, optimal power condition and optimal torque condition, respectively. The responses after 100s are selected as the steady-state response in order to avoid the impact of initial startup stage fluctuation on the evaluation. The steady-state responses of four parameters are calculated into STD to evaluate the output fluctuation and stability, and the numerical value is standardized based on the responses under the baseline controller.
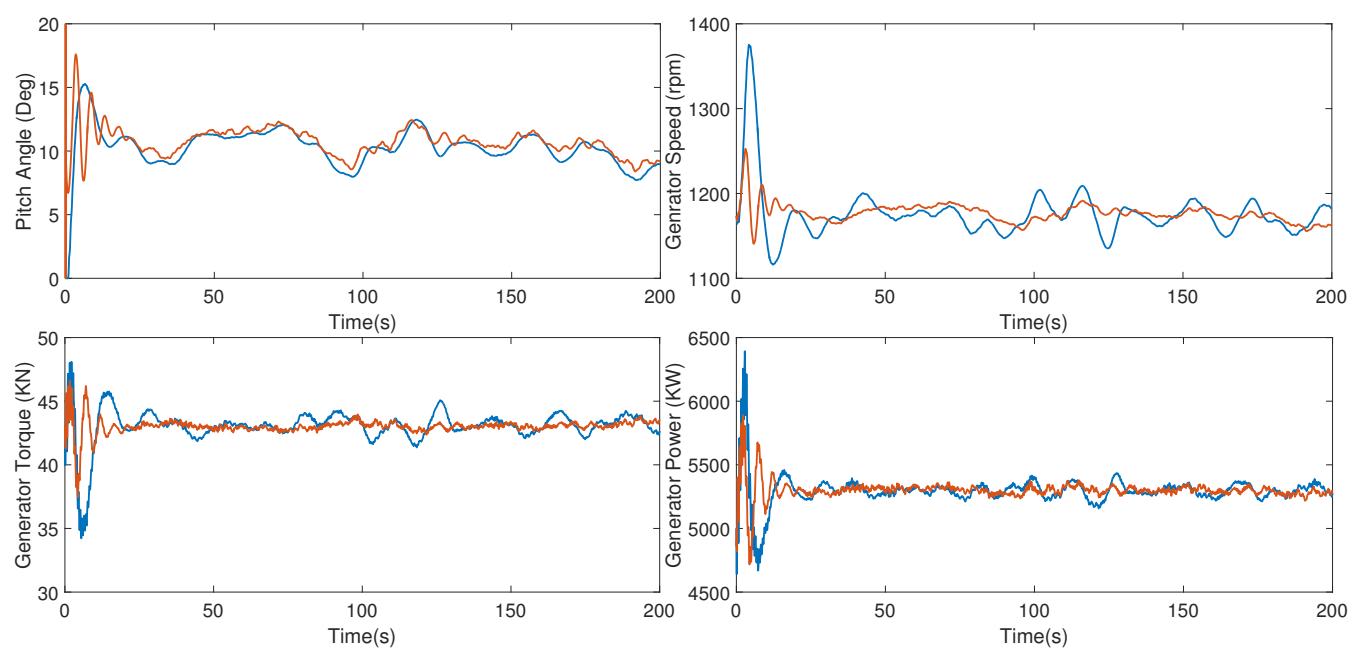

Figure 11: Basic Loads Curves (The blue curves shows the wind turbine responses with the baseline controller, and the orange curves are the responses with the satisfactory solution)

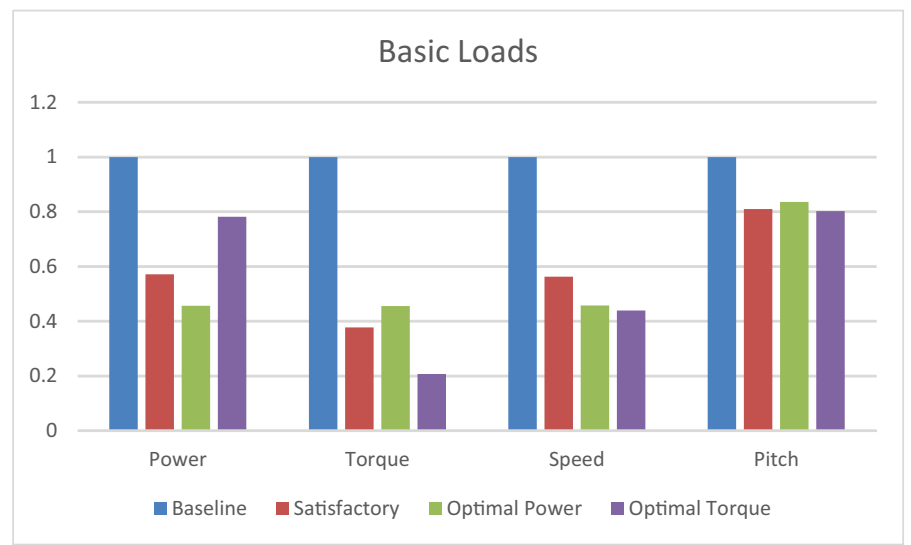

Figure 12: Basic Loads Histogram

In Figure 11, it is obvious that the satisfactory solution achieves better dynamic 
responses in all four analyzed parameters than the baseline controller, the improvement of the generator speed and generator torque fluctuations are significant. The blade pitch moves under the satisfactory solution that the turbulence in the wind speed can be effectively suppressed. The generator power output under the satisfactory solution is also more stable than the baseline controller. In Figure 12, the blue column is taller than other three columns, which shows that a better response can be achieved when poles of the system are placed in either of the three solutions. Besides, the optimal power solution has the lowest generator power STD and the optimal torque solution has the lowest generator torque STD, which is consistent with our discussions in Figure 4. The generator speed STD of the satisfactory solution is higher than the other two solutions, but still has a great influence on the baseline controller. Apart from this, the other three parameters of the satisfactory solution strikes a good balance between the optimal power and torque conditions.

All three driveshaft load responses are plotted in Figure 13. Although it can be found that, the drive shaft loads responses of the satisfactory solution has some improvements than the baseline control loads responses, but the form of responses curves still cannot show the improvement effectively. Moreover, it is difficult to plot all four load curves of one load parameter in one figure. The bending moment on the blade root is positively related to the mechanical stress, and once the mechanical stress exceeds the fatigue limit of the metal, the blade will be damaged. The greater the mechanical stress, or more times the mechanical stress exceeds the fatigue limit, the greater the damage to the blade. The above discussion will be the same on a negative bending moment, which is also a positive parameter but with an opposite direction. Thrust force on the drive shaft is the same. Thus, both mean value and maximum value of the load parameters are calculated for the comparatively overall analysis of the wind turbine loads. The spider diagram is used to show the load comparisons of the baseline control with all three solutions and shown in Figure 14. The specific numerical values of the load parameters are listed in Appendix B. 

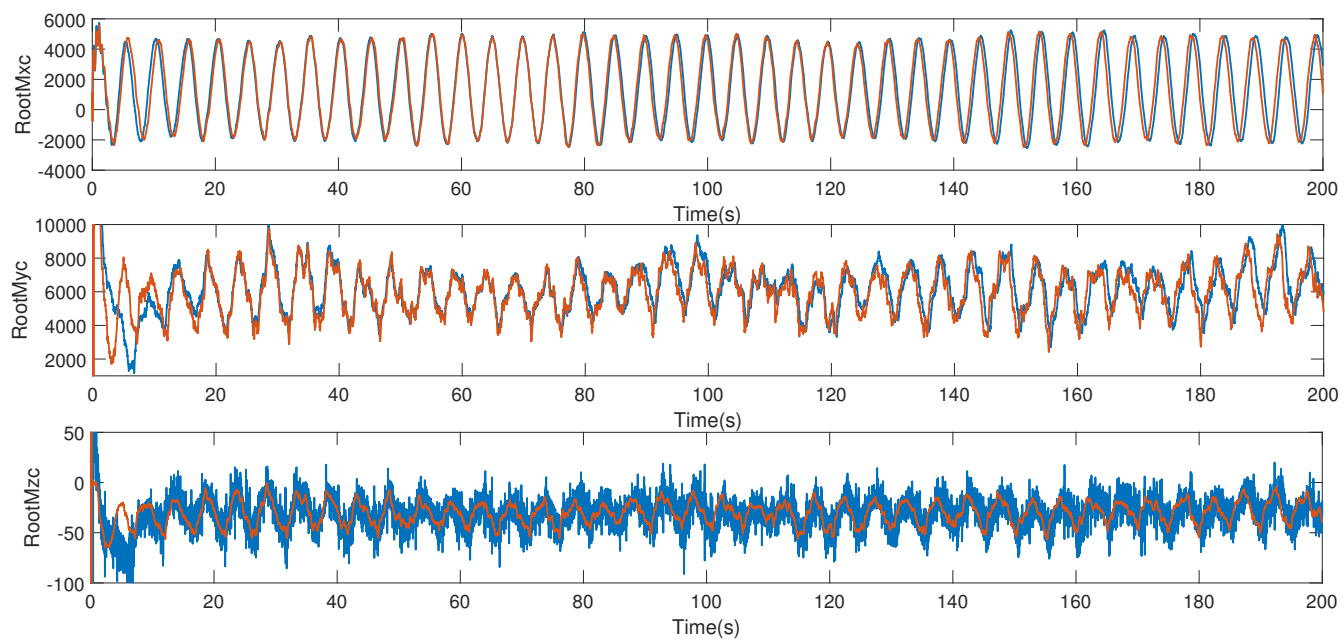

Figure 13: Blade Root Loads (The blue curves are the baseline responses and the orange curves are the satisfactory solution responses)
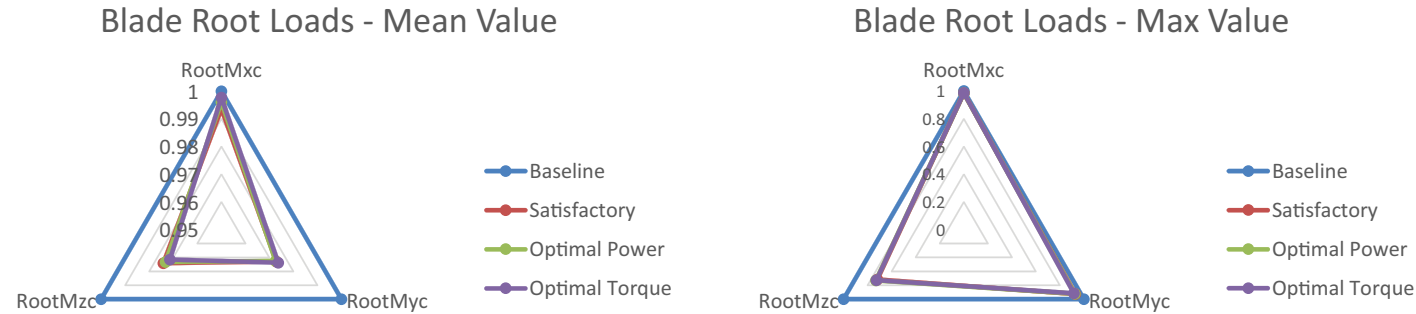

Figure 14: Blade Root Loads Spider Plot

From Table B.4 and B.5, it can be found that the "RootMyc" is the most significant one in three blade root bending moments, which proves that the out-of-plane bending moment has the most great impact on the blade root. Obviously, the optimal torque solution has the smallest max value while the mean values are very close, and the conclusion can be given as: the optimal torque solution is also the optimal blade load solution. In addition, Figures 13 and 14 show that all three solutions achieve better blade load conditions than the baseline controller in both the mean value and max value. Therefore, all three selected solutions are acceptable for the blade load. The response curves and spider diagrams of the drive shaft loads are shown and analyzed as follows. 

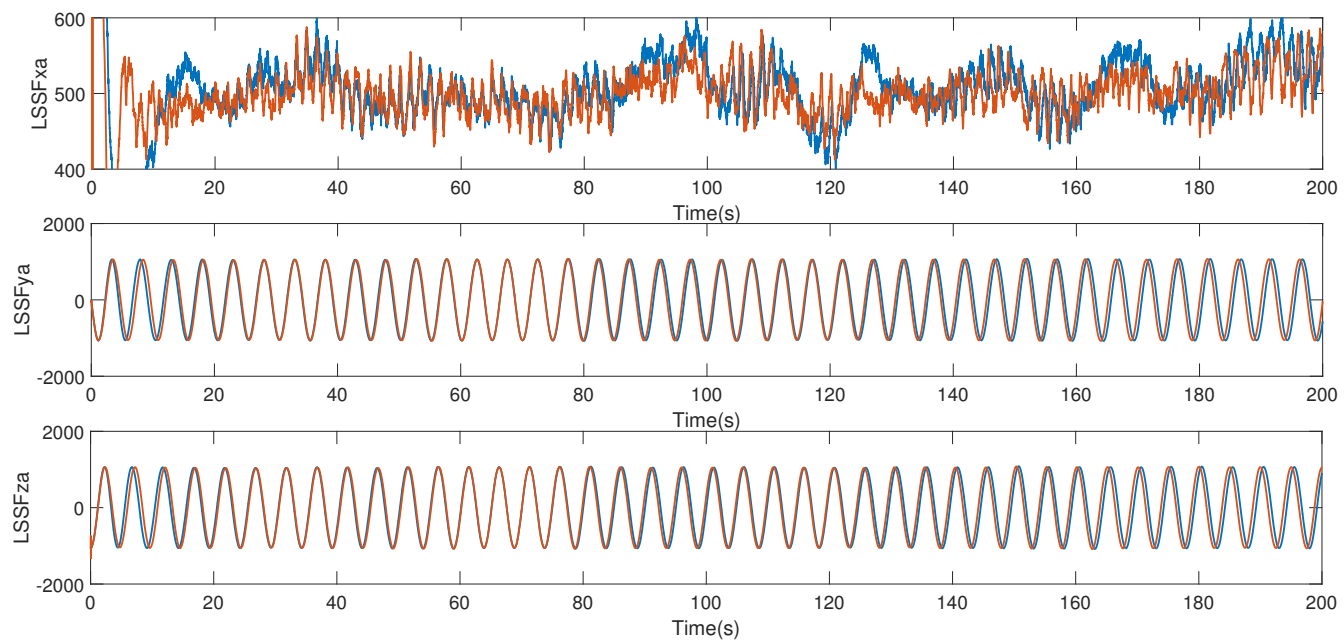

Figure 15: Drive Train Shaft Loads (The blue curves are the baseline responses and the orange curves are the satisfactory solution responses)
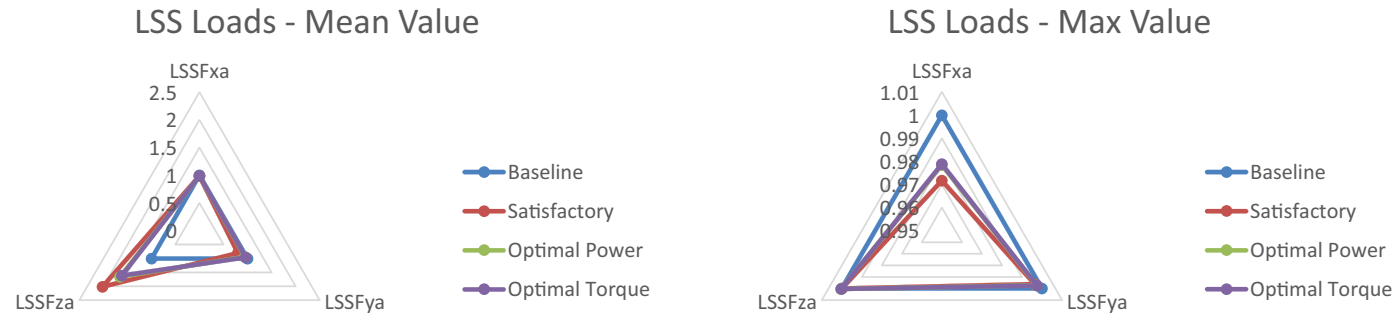

Figure 16: Drive Train Shaft Loads Spider Plot

The first thing to notice in Figure 16 is that, the mean value of "LSSFza" is much bigger in the satisfactory solution than the baseline controller, but it does not have a impact in such situations that the mean value of "LSSFza" is very small, and clearly the max value is slightly reduced with the increase in the mean value. On the other hand, the mean value is very small even the max value is very large, because such thrust force reciprocates between negative and positive, and a small increase in the mean value is obvious if calculated in the magnification, which implies that it is necessary and more meaningful to consider the max value. Numerical results and spider diagrams show that all solutions have better effects on the drive shaft thrust force than the baseline controller, but the difference between solutions are not 
remarkable enough to distinguish which is the best. Although one of the thrust force mean value is increased, the drive shaft loads of three selected solutions still have small improvements and is acceptable.

In summery, the baseline controller is a simple structured, very effective controller, which can be easily applied in the industrial field, but the above comparisons and analysis show that there still exists a big gap between the baseline controller and the weak optimal solutions on the Pareto frontier. The gap can be easily and effectively reduced by the above optimization strategy based on historical data or series of simulations. Both output stability and wind turbine load conditions can be improved significantly through the optimization. Subsequent analysis will compare the simulation results from other perspectives.

\subsection{Eigenvalues Evaluation}

Eigenvalue is one of the inherent attributes of the system, which can reflect the performance of the system and is usually adopted to evaluate the system $[6,7,8,20]$. The participation of the controller will also change the eigenvalues of the closedloop system. Considering both the pitch and generator torque controllers, the wind turbine generator system can be represented as a two-input system and listed in the Appendix. Table 2 shows both the real and imaginary parts of the eigenvalues and the change that happens when different control strategies are adopted.

Table 2: Eigenvalues of the wind turbine system

\begin{tabular}{|l|l|c|c|}
\hline \multicolumn{2}{|c|}{ Systems } & Real & Imag \\
\hline \multirow{2}{*}{ Origion } & $\lambda_{1}$ & -0.157319884 & 0 \\
\cline { 2 - 4 } & $\lambda_{2,3}$ & -0.708718718 & \pm 13.9472861304907 \\
\hline \multirow{2}{*}{ Satisfactory } & $\lambda_{1}$ & -1.382962649 & 0 \\
\cline { 2 - 4 } & $\lambda_{2,3}$ & -0.708140015 & \pm 13.9452367449432 \\
\hline \multirow{2}{*}{ Optimal Power } & $\lambda_{1}$ & -1.812921426 & 0 \\
\cline { 2 - 4 } & $\lambda_{2,3}$ & -0.708160627 & \pm 13.9452325601310 \\
\hline \multirow{2}{*}{ Optimal Torque } & $\lambda_{1}$ & -1.733014965 & 0 \\
\cline { 2 - 4 } & $\lambda_{2,3}$ & -0.708113857 & \pm 13.9452364325443 \\
\hline
\end{tabular}

The wind turbine has a real eigenvalue and a pair of complex eigenvalues with imaginary parts. Once ignoring the effects of zeros, the WTGS can be simplified as a classic three-order transfer function written as:

$$
G(s)=\frac{\omega_{n}^{2} s_{0}}{\left(s+s_{0}\right)\left(s^{2}+2 \zeta \omega_{n} s+\omega_{n}^{2}\right)} .
$$


For the above three-order transfer function, $\left(s+s_{0}\right)$ leads to a negative real eigenvalue, while $\left(s^{2}+2 \zeta \omega_{n} s+\omega_{n}^{2}\right)$ results in a pair of complex eigenvalues. When the input $u(t)$ is a unit step response function and $0<\zeta<1$, output can be transformed by Laplace transform into:

$$
Y(s)=\frac{1}{s}+\frac{A}{s+s_{0}}+\frac{B}{s+\zeta \omega_{n}-j \omega_{n} \sqrt{1-\zeta^{2}}}+\frac{C}{s+\zeta \omega_{n}+j \omega_{n} \sqrt{1-\zeta^{2}}},
$$

wherein

$$
\begin{aligned}
A & =\frac{-\omega_{n}^{2}}{\left(s^{2}-2 \zeta \omega_{n} s+\omega_{n}^{2}\right)}, \\
B & =\frac{s_{0}\left(2 \zeta \omega_{n}-s_{0}\right)-j s_{0}\left(2 \zeta^{2} \omega_{n}-\zeta s_{0}-\omega_{n}\right) / \sqrt{1-\zeta^{2}}}{2\left[\left(2 \zeta^{2} \omega_{n}-\zeta s_{0}-\omega_{n}\right)^{2}+\left(2 \zeta \omega_{n}-s_{0}\right)^{2}\left(1-\zeta^{2}\right)\right]} \\
C & =\frac{s_{0}\left(2 \zeta \omega_{n}-s_{0}\right)+j s_{0}\left(2 \zeta^{2} \omega_{n}-\zeta s_{0}-\omega_{n}\right) / \sqrt{1-\zeta^{2}}}{2\left[\left(2 \zeta^{2} \omega_{n}-\zeta s_{0}-\omega_{n}\right)^{2}+\left(2 \zeta \omega_{n}-s_{0}\right)^{2}\left(1-\zeta^{2}\right)\right]} .
\end{aligned}
$$

Let $b=s_{0} /\left(\zeta \omega_{n}\right)$, then (1) can be transformed by Inverse Laplace transform as:

$y(t)=1+A_{h} e^{-s_{0} t}+2 R e B \cdot e^{-\zeta \omega_{n} t} \cos \left(\omega_{n} t \sqrt{1-\zeta^{2}}\right)-2 \operatorname{ImB} \cdot e^{-\zeta \omega_{n} t} \sin \left(\omega_{n} t \sqrt{1-\zeta^{2}}\right)$,

wherein

$$
\begin{aligned}
A_{h} & =-\frac{1}{b \zeta^{2}(b-2)+1} \\
\operatorname{Re} B & =-\frac{b \zeta^{2}(b-2)}{2\left[b \zeta^{2}(b-2)+1\right]} \\
\operatorname{ImB} & =-\frac{b \zeta\left[\zeta^{2}(b-2)+1\right]}{2\left[b \zeta^{2}(b-2)+1\right] \sqrt{1-\zeta^{2}}} .
\end{aligned}
$$

From the above expression of the classic third-order transfer function, it can be found that the single real eigenvalue has the most important impact on the dynamic response of a third-order system than other eigenvalues. The larger the absolute value of the single real eigenvalue, the faster the decaying of the corresponding response components, conversely slower. In Table 2, the second-order part of the wind turbine model has a small change with different controllers, but the change of the one-order eigenvalue is significant. Eigenvalues of the optimal power solution and the optimal torque solution moving away from the imaginary axis will have faster responses. The optimal power solution has the eigenvalue farthest from the imaginary axis and the 
fastest generator power output response among all the solutions. The eigenvalue of the satisfactory solution move slightly towards the imaginary axis than both optimal solutions, which has lost a small portion of power stability to achieve a balanced condition (between generator power smoothing and generator torque ripple), but its real eigenvalue is still much further than the original wind turbine, for which a fast and stable response can be guaranteed. Unfortunately, it is difficult to calculate the eigenvalues of the wind turbine under the baseline controller, but the results of the eigenvalue analysis of three proposed solutions are consistent with the previous analysis.

\subsection{Frequency Domain Evaluation}

Fast Fourier transformation is adopted to evaluate the response data in the frequency domain. Due to the fact that the three selected solutions have small differences in the frequency domain, the power spectrum is used to evaluate the difference between three selected solutions and only the satisfactory solution is chosen to compare with the baseline controller by fast Fourier transformation. The frequency domain results are shown in Figure 17 and 18.

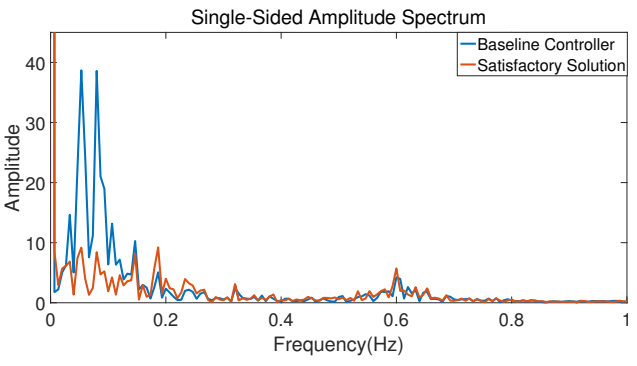

Figure 17: Frequency Domain Evaluation

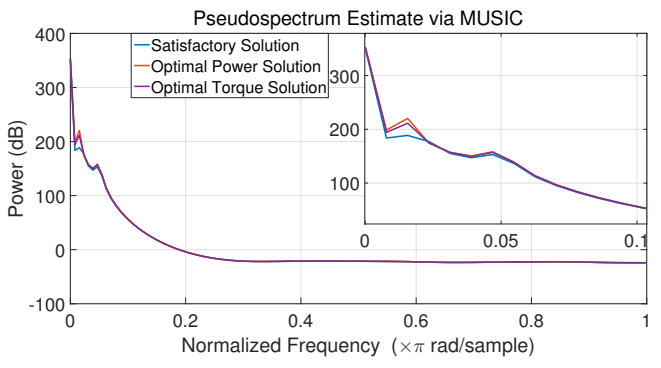

Figure 18: Power Spectrum via MUSIC Algorithm

From Figure 17, it can be found that there exist two peaks in the low-frequency band on the amplitude spectrum of baseline controller, which have been significantly improved by the satisfactory solution. The differences in other frequency segments are quite small, which indicates that the satisfactory solution has great ability to suppress energy fluctuation below $0.2 \mathrm{~Hz}$, i.e., the output power fluctuation at 5-20 seconds scale caused by the wind turbulence can be suppressed effectively. Figure 18 shows that the three selected solutions are very close in the power spectrum. The satisfactory solution has the best power spectral response in detail, and the optimal torque has a middle response between other two solutions in the frequency domain. Although the optimal power solution achieves the best power stability in the time 
domain, but it is still less than the satisfactory solution in the frequency domain. The difference is not so significant but can still be observed.

\section{Coordination Analysis}

A satisfactory solution for torque-pole and pitch-pole are obtained and guarantees better responses than the baseline control strategy. Besides, an optimal power solution and an optimal torque solution are also obtained based on Pareto efficiency. In this section, controller coordination characteristics are discussed based on the response characteristics curve and three selected solutions.

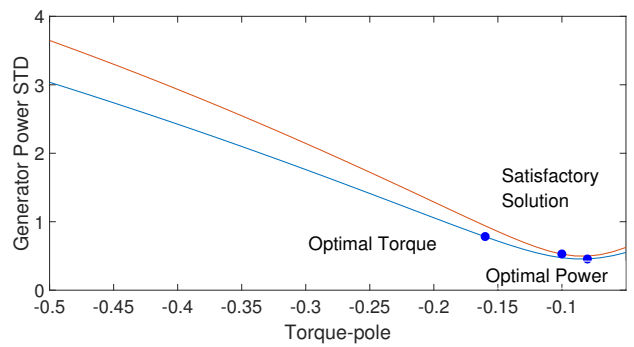

Figure 19: Generator Power STD

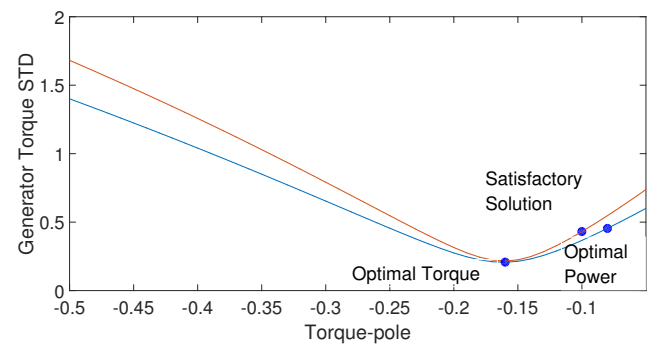

Figure 21: Generator Torque STD

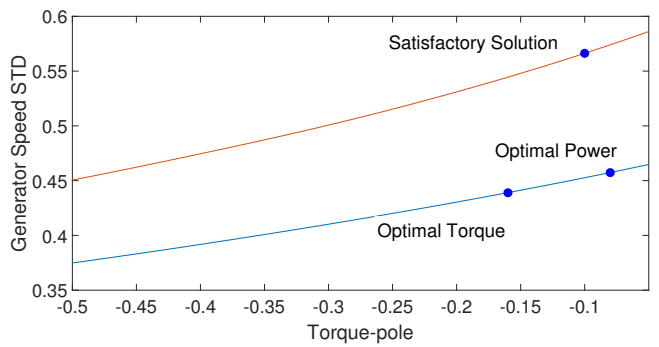

Figure 20: Generator Speed STD

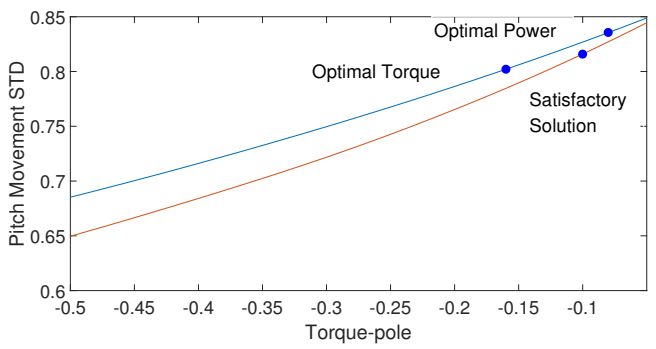

Figure 22: Pitch Movement STD

Clearly, the satisfactory solution reaches a good balance between the two optimal solutions. It should be noticed that the generator speed STD curve has a different y-axis interval with other figures, the difference between solutions seems great but is not significant actually. Apart from this, some other solutions might be needed for different conditions can be summed up from above figures. First, when the generator power stability is the primary consideration during the controller design, the optimal power solution is the best solution, and a similar situation happens to the optimal 
torque one when the generator torque stability is first considered. Meanwhile, the improvement of generator torque stability has a good effect on blade loads, which is one of the most vulnerable components in wind turbines. The optimal torque solution is also the optimal blade load solution. Secondly, the optimal power solution at the expense of the blade pitch frequent actions. In order to decrease the blade pitch actions, increasing the torque pole or decreasing the pitch-pole are both appropriate. It should be noticed that the increase of the torque pole will bring the fluctuation of the generator speed, but the decrease of the pitch-pole can reach relatively less fluctuations of generator speed. Thus, the change of the pitch-pole could be the first choice, but the increase of the torque pole is also needed to coordinate with the pitch-pole of movement. The change of poles positions and corresponding influence is shown in the figure below.

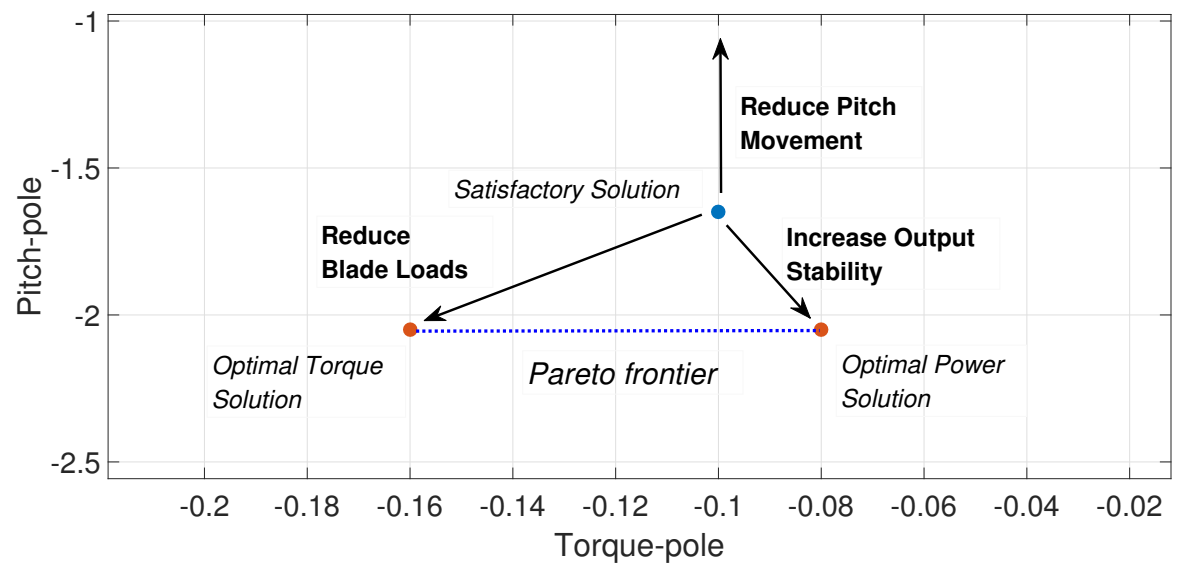

Figure 23: Change of Poles Positions and Corresponding Influence

Additionally, the pole tuning strategy can also be concluded from the above discussions: the pitch-pole can be placed far from the imaginary axis that the system stability is guaranteed, after this, the tuning on the torque-pole should reach a minimum generator power STD. The torque-pole at the current condition is closed to the one in two optimal solutions, and then continue to adjust the pitch-pole. Repeat the adjustment several times when two optimal solutions are obtained. As such, the satisfactory one can be easily obtained to adjust the poles as needed.

Combine all the above discussions, the entire optimization steps can be further summarized as the procedures as shown in Figure 24. 


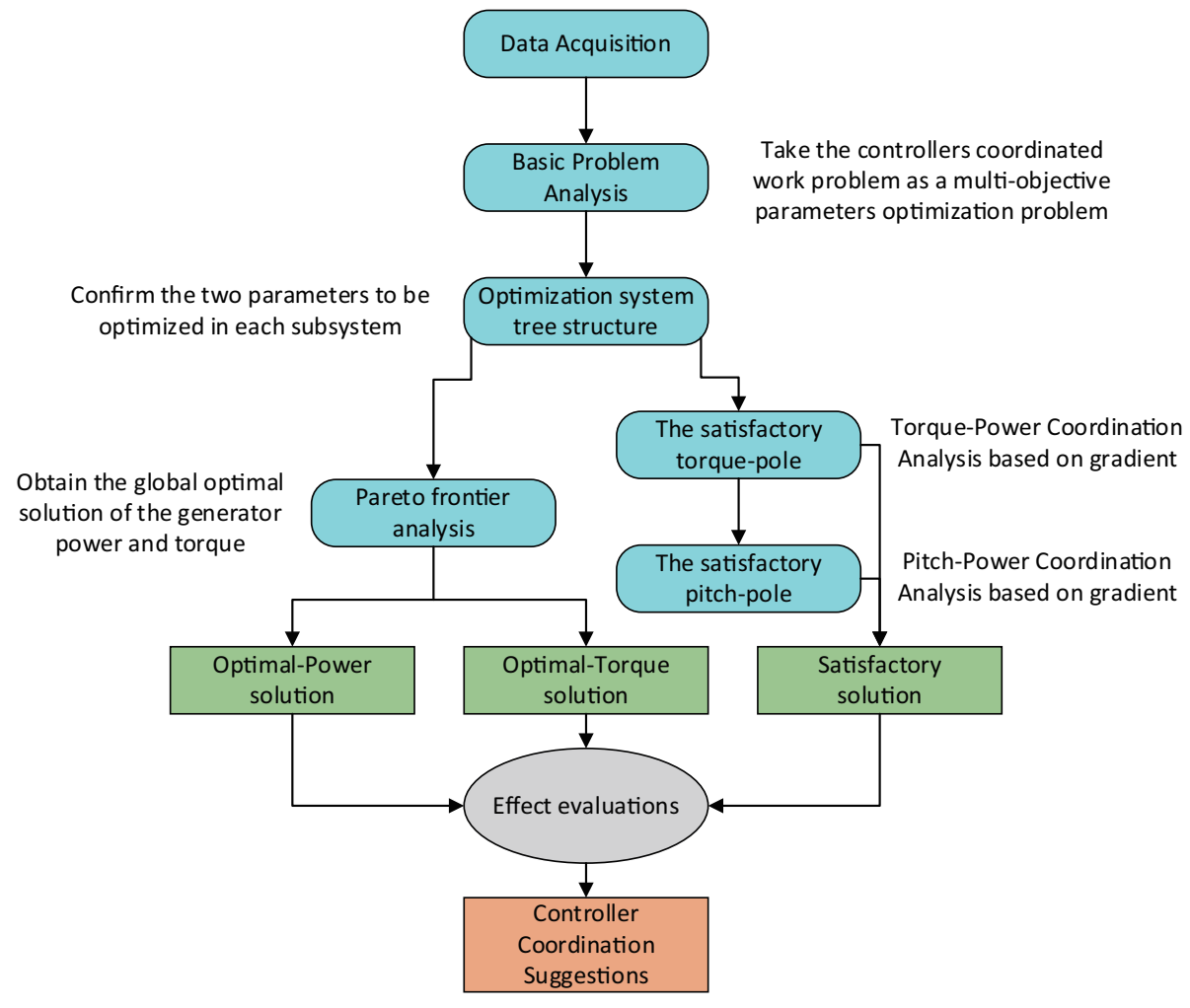

Figure 24: Optimization Flow Chart

\section{Conclusion}

In this paper, the coordination between the blade pitch controller and generator torque \& power controller of WTGS has been analyzed. A two-level parameterscontrollers coordinated optimization system was formed and adopted to solve the optimization problem with the concept of Pareto efficiency. The optimal power solution and optimal torque solution were obtained, then the gradient was calculated to select the satisfactory torque-pole on the Pareto frontier, and the satisfactory pitch-pole on the pitch-power relations. The selected satisfactory poles form the satisfactory solution for the WT. Then all three selected typical solutions were compared with the baseline controller, which shows the difference and improvement in both time domain and frequency domain, and the coordination suggestions have been given based on the results. Further research could be concentrated on the controller coordination and optimization based on the linear-quadratic control and model predictive control. 


\section{Appendix A. WTGS Modelling}

Combining the torque control and pitch control, the unified nonlinear comprehensive model of WTGS can be formulated as:

$$
\left[\begin{array}{c}
\dot{\delta} \\
\dot{\omega}_{r} \\
\dot{\omega}_{g}
\end{array}\right]=\left[\begin{array}{c}
\omega_{r}-\frac{1}{n_{g}} \omega_{g} \\
\frac{1}{J_{r}} T_{r}-\frac{B_{l s}}{J_{r}} \omega_{r}+\frac{B_{l s}}{J_{r} r_{g}} \omega_{g}-\frac{K_{l s}}{J_{r}} \delta \\
\frac{B_{l s}}{J_{g} n_{g}} \omega_{r}-\frac{B_{l s}}{J_{g} n_{g}^{2}} \omega_{g}+\frac{L_{l s}}{J_{g} n_{g}} \delta-\frac{1}{J_{g}} T_{e m}
\end{array}\right] .
$$

From (A.1), the nonlinearity of WTGS concentrates on the rotor torque $T_{r}$, which is a nonlinear function of wind speed $V$, rotor speed $\omega_{r}$ and pitch angle $\beta$, and can be linearized around several operating points. Because the wind speed $V$ can vary with the operating points of WTGS, it can be defined as the varying parameter $r_{t}$ to schedule the whole wind power generation process. Consequently, we select the state variable $x=\left[\delta, \omega_{r}, \omega_{g}\right]^{\prime}$, and set the control variable $u=\left[T_{e m}, \beta\right]^{\prime}$.

For (A.1), define the operating points as $\bar{x}_{i}=\left[\bar{\delta}_{i}, \bar{\omega}_{r i}, \bar{\omega}_{g i}\right]^{\prime}$, where the parameter $i$ is corresponding to the current operating point.For the nonlinear term $T_{r}$ in (A.1), applying the Taylor expansion on the wind speed $V$ and omitting the higher order terms, it can be expressed as:

$$
T_{r}\left(V, \omega_{r}, \beta\right)=T_{r}\left(\bar{V}_{i}, \bar{\omega}_{r i}, \bar{\omega}_{\beta i}\right)+\Gamma_{V}(i) \hat{V}+\Gamma_{\omega}(i) \hat{\omega}_{r}+\Gamma_{\beta}(i) \hat{\beta}
$$

with

$$
\Gamma_{V}(i)=\left.\frac{\partial T_{r}}{\partial V}\right|_{\left(\bar{V}_{i}, \bar{\omega}_{r i}, \bar{\beta}_{i}\right)}, \Gamma_{\omega_{r}}(i)=\left.\frac{\partial T_{r}}{\partial \omega_{r}}\right|_{\left(\bar{V}_{i}, \bar{\omega}_{r i}, \bar{\beta}_{i}\right)}, \Gamma_{\beta}(i)=\left.\frac{\partial T_{r}}{\partial \beta}\right|_{\left(\bar{V}_{i}, \bar{\omega}_{r i}, \bar{\beta}_{i}\right)} .
$$

Herein, the state variables of WTGS can be represented as,

$$
x=\bar{x}+\hat{x},
$$

where $\bar{x}$ is the steady-state operating point, $\hat{x}$ is the deviation from $\bar{x}$ related to the dynamic process. Obviously, we have the dynamic model for each operating region:

$$
\left[\begin{array}{c}
\dot{\hat{\delta}} \\
\dot{\hat{\omega}}_{r} \\
\dot{\hat{\omega}}_{g}
\end{array}\right]=\left[\begin{array}{c}
\hat{\omega}_{r}-\frac{1}{n_{g}} \hat{\omega}_{g} \\
\frac{1}{J_{r}}\left(\Gamma_{V}(i) \hat{V}+\Gamma_{\omega_{r}}(i) \hat{\omega}_{r}+\Gamma_{\beta}(i) \hat{\beta}\right)-\frac{B_{l s}}{J_{r}} \hat{\omega}_{r}+\frac{B_{l s}}{J_{r} n_{g}} \hat{\omega}_{g}-\frac{K_{l s}}{J_{r}} \hat{\delta} \\
\frac{B_{l s}}{J_{g} n_{g}} \hat{\omega}_{r}-\frac{B_{l s}}{J_{g} n_{g}^{2}} \hat{\omega}_{g}+\frac{K_{l s}}{J_{g} n_{g}} \hat{\delta}^{-1} \frac{1}{J_{g}} \hat{T}_{e m}
\end{array}\right] .
$$

Based on the above discussion, by applying the Taylor expansion around the operating point $\bar{x}_{i}$, and setting $\hat{x}=\left[\hat{\delta}, \hat{\omega}_{r}, \hat{\omega}_{g}\right]^{\prime}$, and the WTGS output as $\hat{y}=\left[\hat{\omega}_{g}\right]^{\prime}$, the system (A.1) can be linearized into a linear system with the following form:

$$
\left\{\begin{array}{l}
\dot{\hat{x}}=\hat{A}_{i} \hat{x}+\hat{B}_{i} \hat{u}+\hat{B}_{V i} \hat{V} \\
\hat{y}=\hat{C} \hat{x}
\end{array}\right.
$$


where

$$
\begin{gathered}
\hat{A}_{i}=\left[\begin{array}{ccc}
0 & 1 & -\frac{1}{n_{g}} \\
-\frac{K_{l s}}{J_{r}} & \frac{\Gamma_{\omega_{r}}(i)}{J_{r}}-\frac{B_{l s}}{J_{r}} & \frac{B_{l s}}{J_{r} n_{g}} \\
\frac{K_{l s}}{J_{g} n_{g}} & \frac{B_{l s}}{J_{g} n_{g}} & -\frac{B_{l s}}{J_{g} n_{g}^{2}}
\end{array}\right], \hat{B}_{i}=\left[\begin{array}{cc}
0 & 0 \\
0 & \frac{\Gamma_{\beta}(i)}{J_{r}} \\
-\frac{1}{J_{g}} & 0
\end{array}\right], \\
\hat{B}_{V i}=\left[\begin{array}{c}
0 \\
0 \\
\frac{\Gamma_{V}(i)}{J_{r}}
\end{array}\right], \hat{C}=\left[\begin{array}{lll}
0 & 0 & 1
\end{array}\right] .
\end{gathered}
$$

The poles placement method or other control strategies should be applied to the multiple-input single-output (MISO) model in the system (A.1), but it can be also split into two single-input single-output (SISO) system for the pitch control subsystem and torque control subsystem separately when ignoring the effects of wind turbulence. Take the control variable into two parts, pitch control variable $\hat{u_{1}}=[\beta]^{\prime}$ and torque control variable $\hat{u_{2}}=\left[T_{e m}\right]^{\prime}$, the system (A.3) can be divided as:

$$
\left\{\begin{array}{l}
\dot{\hat{x}}=\hat{A}_{i} \hat{x}+\hat{B}_{1 i} \hat{u}_{1}+\hat{B}_{2 i} \hat{u}_{2}, \\
\hat{y}=\hat{C} \hat{x}
\end{array}\right.
$$

where

$$
\hat{B}_{1 i}=\left[\begin{array}{c}
0 \\
\frac{\Gamma_{\beta}(i)}{J_{r}} \\
0
\end{array}\right], \hat{B}_{2 i}=\left[\begin{array}{c}
0 \\
0 \\
-\frac{1}{J_{g}}
\end{array}\right],
$$

The model of the blade pitch controller and generator torque \& power controller is shown below.

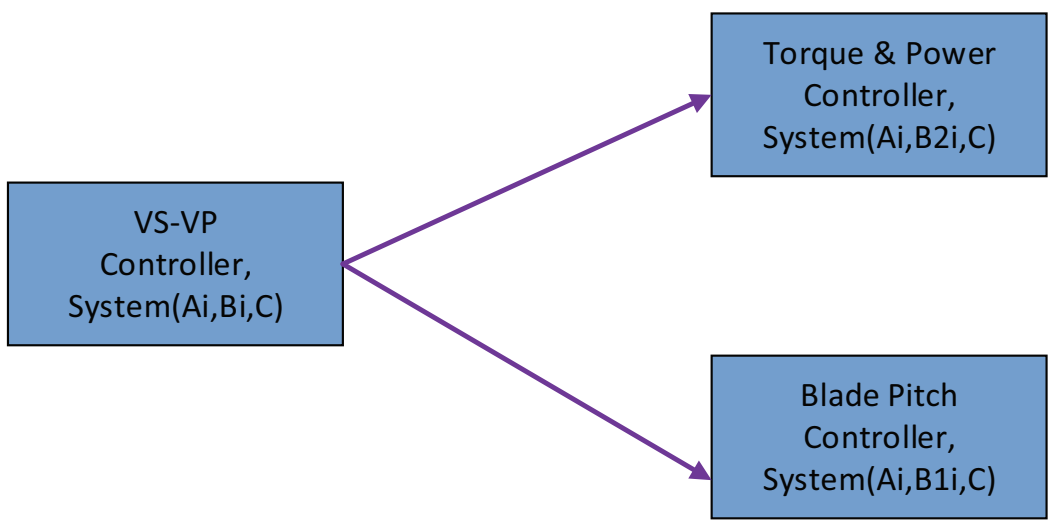

Figure A.25: Controller Models 


\section{Appendix B. Tables}

Table B.3: Basic Loads - STD

\begin{tabular}{|l|l|l|l|l|}
\hline Basic Loads & Power & Torque & Speed & Pitch \\
\hline Baseline & 1 & 1 & 1 & 1 \\
\hline Satisfactory & 0.51248202 & 0.337796738 & 0.496627859 & 0.823884459 \\
\hline Optimal Power & 0.415591417 & 0.400927649 & 0.404549582 & 0.850725769 \\
\hline Optimal Torque & 0.674863518 & 0.190774019 & 0.389403324 & 0.819020056 \\
\hline
\end{tabular}

Table B.4: Blade Root Loads - Mean Value

\begin{tabular}{|l|l|l|l|}
\hline Blade-Mean & RootMxc & RootMyc & RootMzc \\
\hline Baseline & 1350.264817 & 6224.87278 & 30.50432075 \\
\hline Satisfactory & 1341.538116 & 6055.924381 & 29.71491544 \\
\hline Optimal Power & 1344.837877 & 6054.190179 & 29.68779313 \\
\hline Optimal Torque & 1347.15075 & 6061.061472 & 29.63268369 \\
\hline
\end{tabular}

Table B.5: Blade Root Loads - Max Value

\begin{tabular}{|l|l|l|l|}
\hline Blade-Max & RootMxc & RootMyc & RootMzc \\
\hline Baseline & 5248.788086 & 10157.74414 & 19.9146328 \\
\hline Satisfactory & 5168.694824 & 9434.576172 & 4.125574589 \\
\hline Optimal Power & 5173.102051 & 9388.236328 & 4.675378323 \\
\hline Optimal Torque & 5179.195313 & 9313.331055 & 5.689994335 \\
\hline
\end{tabular}

Table B.6: Drive Shaft Loads - Mean Value

\begin{tabular}{|l|l|l|l|}
\hline DT-Mean & LSSFxa & LSSFya & LSSFza \\
\hline Baseline & 509.3474252 & 7.444877874 & 3.665385889 \\
\hline Satisfactory & 503.7592906 & 5.952962272 & 7.409603249 \\
\hline Optimal Power & 503.7135445 & 7.03244494 & 6.07221951 \\
\hline Optimal Torque & 504.1744214 & 7.143466051 & 5.923596697 \\
\hline
\end{tabular}


Table B.7: Drive Shaft Loads - Max Value

\begin{tabular}{|l|l|l|l|}
\hline DT-Max & LSSFxa & LSSFya & LSSFza \\
\hline Baseline & 603.1212769 & 1076.631348 & 1084.646362 \\
\hline Satisfactory & 586.0315552 & 1071.898804 & 1080.150635 \\
\hline Optimal Power & 590.0865479 & 1072.210693 & 1080.612427 \\
\hline Optimal Torque & 590.3475952 & 1072.603271 & 1080.824951 \\
\hline
\end{tabular}

\section{References}

[1] Kumar, D., \& Chatterjee, K. (2016). A review of conventional and advanced MPPT algorithms for wind energy systems. Renewable and Sustainable Energy Reviews, 55, 957-970.

[2] Jha, A. R. (2010). Wind turbine technology. CRC press.

[3] Senjyu, T., Sakamoto, R., Urasaki, N., Funabashi, T., Fujita, H., \& Sekine, H. (2006). Output power leveling of wind turbine generator for all operating regions by pitch angle control. IEEE Transactions on Energy conversion, 21(2), 467-475.

[4] El-Tous, Y. (2008). Pitch angle control of variable speed wind turbine. American Journal of Engineering and Applied Sciences, 1(2), 118-120.

[5] Jonkman, J., Butterfield, S., Musial, W., \& Scott, G. (2009). Definition of a 5-MW reference wind turbine for offshore system development (No. NREL/TP500-38060). National Renewable Energy Laboratory (NREL), Golden, CO..

[6] Eisa, S. A., Wedeward, K., \& Stone, W. (2016). Sensitivity analysis of a type-3 DFAG wind turbine's dynamics with pitch control. Green Energy and Systems Conference. IEEE.

[7] Eisa, S. A., Stone, W., \& Wedeward, K. (2017). Mathematical Modeling, Stability, Bifurcation Analysis, and Simulations of a Type-3 DFIG Wind Turbine's Dynamics with Pitch Control. Green Technologies Conference. IEEE.

[8] Eisa, S. A., Wedeward, K., \& Stone, W. (2017). Time Domain Study of a Type3 DFIG Wind Turbine's Dynamics: Q Drop Function Effect and Attraction vs Control Limits Analysis. Green Technologies Conference. IEEE.

[9] Khalil, H. K. (1996). Noninear Systems. Prentice-Hall, New Jersey, 2(5), 5-1. 
[10] Yin, X. X., Lin, Y. G., Li, W., Gu, Y. J., Lei, P. F., \& Liu, H. W. (2015). Adaptive back-stepping pitch angle control for wind turbine based on a new electro-hydraulic pitch system. International Journal of Control, 88(11), 23162326 .

[11] Hwas, A., \& Katebi, R. (2012). Wind turbine control using PI pitch angle controller. IFAC Proceedings Volumes, 45(3), 241-246.

[12] Abdin, E. S., \& Xu, W. (2000). Control design and dynamic performance analysis of a wind turbine-induction generator unit. IEEE Transactions on Energy Conversion, 15(1), 91-96.

[13] Liu, J., Meng, H., Hu, Y., Lin, Z., \& Wang, W. (2015). A novel MPPT method for enhancing energy conversion efficiency taking power smoothing into account. Energy Conversion and Management, 101, 738-748.

[14] Wright, A. D. (2004). Modern control design for flexible wind turbines (No. NREL/TP-500-35816). National Renewable Energy Lab., Golden, CO (US).

[15] Lin, Z., Liu, J., Zhang, W., \& Niu, Y. (2016). Regional pole placement of wind turbine generator system via a Markovian approach. IET Control Theory \& Applications, 10(15), 1771-1781.

[16] Hassan, H. M., ElShafei, A. L., Farag, W. A., \& Saad, M. S. (2012). A robust LMI-based pitch controller for large wind turbines. Renewable energy, 44, 63-71.

[17] Boukhezzar, B., \& Siguerdidjane, H. (2011). Nonlinear control of a variablespeed wind turbine using a two-mass model. IEEE Transactions on Energy Conversion, 26(1), 149-162.

[18] Torchani, B., Sellami, A., \& Garcia, G. (2016). Variable speed wind turbine control by discrete-time sliding mode approach. ISA Transactions, $62,81-86$.

[19] Lin, Z., Liu, J., \& Niu, Y. (2017). Dynamic response regulation of non-linear feedback linearised wind turbine using a two-mass model. IET Control Theory \& Applications, 11(6), 816-826.

[20] Eisa, S. A., Stone, W., \& Wedeward, K. (2017). Mathematical analysis of wind turbines dynamics under control limits: boundedness, existence, uniqueness, and multi time scale simulations. International Journal of Dynamics and Control(1), $1-21$. 
[21] Zhu, M., Liu, J., Lin, Z., \& Meng, H. (2016). Mixed $H_{2} / H_{\infty}$ pitch control of wind turbine generator system based on global exact linearization and regional pole placement. International Journal of Machine Learning \& Cybernetics, 7(5), $1-10$.

[22] Cui, Y., Geng, Z., Zhu, Q., \& Han, Y. (2017). Multi-objective optimization methods and application in energy saving. Energy.

[23] Pratama, Y. W., Purwanto, W. W., Tezuka, T., McLellan, B. C., Hartono, D., Hidayatno, A., \& Daud, Y. (2017). Multi-objective optimization of a multiregional electricity system in an archipelagic state: The role of renewable energy in energy system sustainability. Renewable and Sustainable Energy Reviews, 77, 423-439.

[24] Odgaard, P. F., Larsen, L. F., Wisniewski, R., \& Hovgaard, T. G. (2016). On using pareto optimality to tune a linear model predictive controller for wind turbines. Renewable Energy, 87, 884-891.

[25] Odgaard, P. F. (2015, July). On usage of pareto curves to select wind turbine controller tunings to the wind turbulence level. In Control Conference (ECC), 2015 European (pp. 1534-1539). IEEE.

[26] Walatka, P. P., Clucas, J., McCabe, R. K., Plessel, T., Potter, R., \& Cooper, D. M. (1994). FAST user guide.

[27] Boyd, S., \& Vandenberghe, L. (2004). Convex Optimization. Cambridge: Cambridge University Press.

[28] Bossanyi, E. A. (2003). Wind turbine control for load reduction. Wind Energy, 6(3), 229244

[29] Han, B., Zhou, L., Yang, F., \& Xiang, Z. (2016). Individual pitch controller based on fuzzy logic control for wind turbine load mitigation. IET Renewable Power Generation, 10(5), 687-693. 